\title{
ESTIMACIÓN DE LA RELACIÓN ENTRE EL NIVEL DE PRECIOS Y LA TASA DE CAMBIO PARA COLOMBIA (1991-2006), MEDIANTE EL FILTRO DE KALMAN*
}

\author{
Fernando Chavarro Miranda** \\ Manfred Grautoff Laverde ${ }^{* * *}$ \\ Erika Viviana Mancipe Moncada***
}

Recibido: julio 12 de 2011 • Aceptado: mayo 16 de 2012

\section{RESUMEN}

Las fluctuaciones de la tasa de cambio se intensificaron en el período 1991-2006. La crisis económica de 1998 giró en torno a las devaluaciones de las monedas que se encontraban bajo tipos de cambio semirrígidos. Esta alteración debería haber corregido los precios de forma significativa, así el nivel general de precios variaba de forma proporcional a la corrección de la tasa de cambio, pero la evidencia sugiere que este fenómeno disminuyó en la última etapa. El objetivo de este artículo es emplear el Filtro de Kalman para capturar la formación de expectativas temporales entre el tipo de cambio y el nivel de precios de la economía. Este ejercicio permite evidenciar que para Colombia en el período 1991-2006 la influencia de la tasa de cambio sobre el nivel de precios se ha reducido de forma sensible, lo que implica que la estimación de la inflación objetivo por parte del Banco Central debe incorporar este hecho para lograr una mayor precisión.

\section{PALABRAS CLAVE}

Inflación, política monetaria, régimen cambiario, efecto transferencia, filtro de Kalman.

\section{CLASIFICACIÓN JEL}

E52, F31, F41, C59

\section{CONTENIDO}

Introducción, 1. Aspectos teóricos; 2. Revisión bibliográfica; 3. La política monetaria en Colombia; 4. Análisis empírico; 5. Conclusiones; Bibliografía.

Este artículo de investigación es producto del proyecto de investigación de "Teorías del desarrollo", adscrito a la de la línea de investigación "Teoría y Política Monetaria", desarrollado durante el período de 2008 a 2011, investigación financiada por la Facultad de Ciencias Económicas, Administrativas y Contables de la Universidad Libre, Grupo de investigación Desarrollo y Sociedad, Universidad Libre de Colombia, Bogotá, Colombia

•* Economista, Universidad de La Salle, Bogotá, Colombia. Especialista en Investigación y Docencia, Universidad Santo Tomás, Bogotá, Colombia. Especialista en Finanzas, Universidad del Rosario, Bogotá, Colombia. Magíster en Economía, Universidad de Los Andes, Bogotá, Colombia. Investigador, Grupo de Desarrollo y Sociedad, Universidad Libre, Bogotá, Colombia. Correo electrónico: fchavarr@uniandino.com.co.

** Economista y Magíster Economía, Universidad de los Andes, Bogotá, Colombia. Magíster en Defensa y Seguridad Nacionales, Escuela Superior de Guerra, Bogotá, Colombia. Investigador, Grupo de Desarrollo y Sociedad, Universidad Libre, Bogotá, Colombia. Correo electrónico: me.grautoff366@uniandes.edu.co.

*** Economista, Escuela Colombiana de Ingeniería Julio Garavito, Bogotá, Colombia. Consultor Técnico Plus Regalías Directas- Subdirección de Control y Vigilancia, Departamento Nacional de Planeación, Bogotá, Colombia. Correo electrónico: erika.viviana@ujaveriana.edu.co. 


\title{
ESTIMATING THE RELATION BETWEEN PRICE LEVELS AND EXCHANGE RATE FOR COLOMBIA (1991-2006) USING KALMAN'S FILTER
}

\section{ABSTRACT}

Fluctuations in the exchange rate intensified between 1991-2006, the economic crisis of 1998 revolved around currency devaluations that were under semi-fixed exchange rate regimes. This alteration should have corrected prices significantly making the general level of prices vary proportionally to the exchange rate correction but the evidence suggests that this phenomenon change in the last year. The goal of this paper is to use Kalman's filter to capture the formation of temporal expectations between the exchange rate and the price level of the economy. This exercise evidence that between 1991-2006, Colombia reduced the influence of the exchange rate over the price level, wich implies that the estimation of the target inflation by the Central Bank must incorporate this fact in order to achieve a higher precision.

\section{KEY WORDS}

Inflation, monetary policy, exchange rate regime, transfer effect, Kalman’s filter

\section{JEL CLASSIFICATION}

\author{
E52, F31, F41, C59
}

\section{CONTENT}

Introduction, 1. Theoretical aspects; 2. Bibliographical review; 3. Monetary policy in Colombia;

4. Empiric analysis; 5. Conclusions; Bibliography.

\section{ESTIMAÇÃO DA RELAÇÃO ENTRE O NIVEL DE PREÇÕS E A TAXA DE CAMBIO PARA COLÔMBIA (1991-2006), MEDIANTE 0 FILTRO DE KALMAN}

\section{RESUMO}

As flutuações da taxa de cambio se intensificaram no período 1991-2006, a crise econômica de 1998, girou em torno as desvalorizações das moedas que se encontravam sob tipos de cambio semirrígidos, esta alteração deveria ter corregido os preços de forma significativa, assim o nível geral de preços variava de forma proporcional à correção da taxa de cambio, mas a evidencia sugere que este fenómeno diminuiu na última etapa. O objetivo deste artigo é empregar o Filtro de Kalman para capturar a formação de expectativas temporais entre o tipo de cambio e o nível de preços da economia. Este exercício permite evidenciar que para Colômbia no período 1991-2006 a influencia da taxa de cambio sobre o nível de preços tem-se reduzido de forma sensível, o que implica que a estimação da inflação objetivo por parte do Banco Central deve incorporar este fato para conseguir uma maior precisão.

\section{PALAVRAS-CHAVE}

Inflação, política monetária, régime cambiário, efeito transferência, filtro de Kalman.

\section{CLASSIFICAÇÃO JEL}

\author{
E52, F31, F41, C59
}

\section{CONTEUDO}

Introdução, 1. Aspectos teóricos; 2. Revisão bibliográfica; 3. A política monetária em Colômbia; 4. Análise empírica; 5. Conclusões; Bibliografia. 
Estimación de la relación entre el nivel de precios y la tasa de cambio para Colombia (1991-2006), mediante el filtro de Kalman

\section{INTRODUCCIÓN}

Las fluctuaciones de la tasa de cambio se han intensificado entre la década de 1990 y el año 2011; así los tipos de cambio fijo no resisten los ataques especulativos. Esto quedó evidenciado en la crisis de Asia 1997 tal lo expresa Krugman (1999, p. 156), este tipo de regímenes cambiarios controlados se derrumbaron. Latinoamérica sufrió un efecto de contagio donde el mecanismo de transmisión de la tasa de cambio arrojó al continente a una crisis generalizada, que desencadenó la alteración social y política de Venezuela, Brasil, Argentina, Ecuador y México.

El hecho estilizado que mostraba correlación proporcional entre nivel de precios y tasa de cambio ha disminuido de forma significativa, a causa de la integración de los mercados, la competencia internacional, la elevación del grado de apertura de las naciones y el modelo de inflación objetivo impuesto por el mundo desarrollado. Afirma Taylor (2000) que el paso a un régimen monetario con información perfecta causa que los agentes generen expectativas ante choques en la tasa de cambio, que solo se captura en forma transitoria; este fenómeno se denomina Pass Through (PT).

Colombia atravesó por cambios dentro de su estructura económica en la década de 1990 como fueron la desgravación arancelaria, la independencia del Banco de la República incorporada en la Constitución de 1991, y la liberalización del régimen cambiario, que afectaron el desempeño macroeconómico de este país, y que alteraron la relación entre el tipo de cambio y la inflación.

Una tendencia decreciente del efecto PT puede influenciar las proyecciones que realicen los bancos centrales respecto al comportamiento futuro de la inflación; este trabajo estima la relación entre la fluctuación de la tasa de cambio y la inflación esperada, lo que permite proporcionar una herramienta de análisis sobre la dinámica de la inflación.

El objetivo del presente artículo es demostrar que el efecto de la tasa de cambio sobre el nivel de precios ha disminuido y, por lo tanto, la modelación de la inflación objetivo debe tener en cuenta este cambio estructural, para proyectar con mayor calidad el dato de inflación que se fija a comienzos de año.

Con el propósito de demostrar esta situación se construye el filtro de Kalman; este permite determinar el coeficiente PT canal de transmisión entre el tipo de cambio y nivel de precios; en este orden se realizan las estimaciones que incorporan este proceso, lo que permite capturar el cambio a través del tiempo sobre la política cambiaria y monetaria. Se elaboran dos estimaciones con dos variables dependientes diferentes: índice de precios al consumidor (IPC) e índice de precios al productor (IPP), con el propósito de constatar si existe diferencia en el coeficiente 
de PT al utilizar distintos indicadores de inflación, lo que permite establecer una medida de validación del modelo.

Los resultados que arroja la evidencia empírica indican que el efecto de la tasa de cambio sobre el nivel de precios se reduce entre los años 1999-2006 debido al cambio de régimen. Así se demuestra que la hipótesis de Taylor que afirma que existe una baja relación entre expectativas y regímenes cambiarios flexibles; por lo tanto, este argumento es válido para el caso de Colombia.

El presente trabajo se divide en seis secciones, que incluyen esta introducción. En la primera sección se exponen los argumentos teóricos del efecto PT, en el segundo apartado se revisa la literatura sobre los distintos enfoques de estudio de PT, en la tercera sección se presenta un análisis de la política monetaria en Colombia, en la cuarta parte estima un modelo para la economía colombiana entre 1991-2006; en la quinta parte se presentan las conclusiones.

\section{ASPECTOS TEÓRICOS}

\subsection{Efecto transferencia en tasa de cambio}

La ley del precio único establece que ante una variación en la tasa de cambio, los precios cambian en igual proporción. Este es el denominado efecto Pass Through, el cual muestra el grado de reacción de los precios ante movimientos en la tasa de cambio nominal.

$\mathrm{P}=\mathrm{E}^{*} \mathrm{P}^{*}$

Donde

P: precios internos

$\mathrm{P}^{*}$ : precios externos

E: tasa de cambio nominal

Si la ley del precio único se cumple, se obtendrá transmisión completa de la variación de la tasa de cambio a los precios; debido a competencia imperfecta, dicha ley no se cumple. Las causas para que no aplique la ley del precio único son:

- Barreras naturales al comercio

- Barreras artificiales

- No todos los bienes se transan en los mercados mundiales

- La construcción del índice de precios local no es igual al índice de precios mundial

- Competencia monopolística

- Diferencias tecnológicas entre países

- Asimetrías de información 


\subsection{Etapas de transmisión}

Por medio de los mecanismos de transmisión monetaria, las acciones de política monetaria influyen sobre las variables económicas como la inflación y el nivel de producción. Existen tres canales mediante los cuales las medidas tomadas por la autoridad monetaria tienen efectos sobre el nivel de precios.

1. Canal demanda: si las tasas de interés aumentan, los agentes prefieren ahorrar, aplazar consumo. Este decrecimiento en el nivel de demanda lleva a que los precios caigan y de este modo, el nivel de inflación decrece.

2. Canal de expectativas: cuando las autoridades monetarias elevan las tasas de interés y sus acciones gozan de credibilidad y confianza entre los agentes, esta alza provoca que las expectativas de inflación se reduzcan y de este modo el nivel de inflación.

3. Tasa de cambio: un alza de la tasa de interés aprecia el tipo de cambio nominal, la demanda por productos importados cae, de esta forma se contrae la inflación de bienes importados y, por tanto, se reduce el nivel de precios en la economía.

Una depreciación de la tasa de cambio se transmite en dos etapas:

En la primera aumentan los precios de importación. Esto lleva a que el ingreso promedio de las firmas importadoras aumente, pero decrezca la cantidad demandada. El incremento del beneficio dependerá de la elasticidad de la demanda (si la curva de demanda presenta una elasticidad que tiende a cero, aumentan los beneficios para la firma) y del poder de mercado que tengan las firmas en el país importador.

En la segunda etapa, la variación de precios de bienes importados afecta los precios de los bienes domésticos por medio de tres canales:

1. Aumento de costos de producción de los bienes nacionales que requieren insumos importados; el precio final de los bienes se ve afectado por un aumento en sus costos de producción.

2. Consumo de bienes importados: el efecto final en el nivel de precios depende del grado de apertura del país y del peso que tengan los bienes importados en la canasta familiar.

3. Estímulos en la demanda de bienes nacionales: en términos relativos, los bienes importados suben de precio con respecto a los bienes nacionales, así la demanda de productos nacionales aumenta, lo que permite elevar el nivel de precios. Este fenómeno se produce en la medida en que los bienes importados tengan bienes sustitutos nacionales. 
Figura 1. Etapas del efecto Transferencia en tasa de cambio

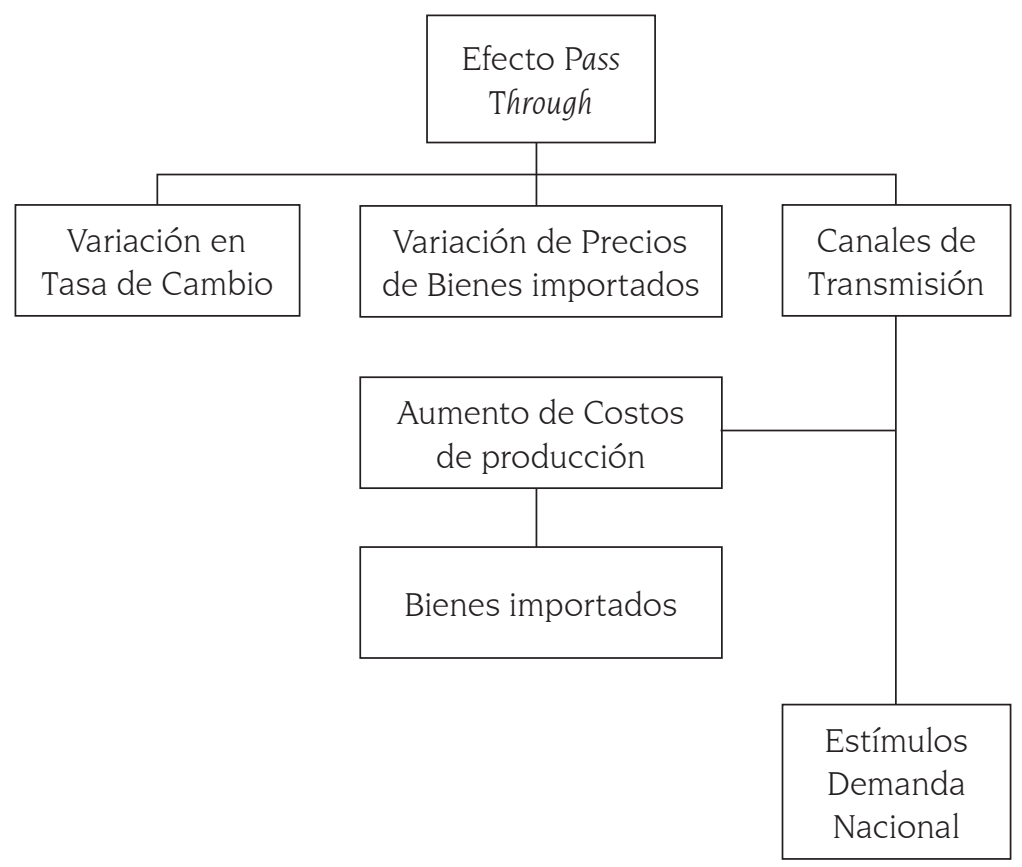

Fuente: construcción propia

\subsection{Variables que afectan el grado de transferencia de la tasa de cambio}

El debate acerca de las causas del bajo grado de PT se ha evidenciado tanto en países desarrollados como en países en vía de desarrollo. Así se han generado dos líneas de investigación que analizan el grado de transmisión de los efectos de la tasa de cambio al nivel de precios:

Linea de estudios microeconómicos: se concentra en la estructura de mercado y la teoría de organización industrial. De este modo, el grado de transferencia es bajo debido a las imperfecciones en los mercados de bienes heterogéneos y plena sustitución entre ellos. Los factores que pueden influenciar el grado el coeficiente PT según esta corriente son:

- La estructura de los mercados y su grado de concentración Krugman (1987, p. 37).

- El grado de homogeneidad y sustitución entre los bienes y la participación de las firmas extranjeras con respecto a los competidores internos en determinado bien (Dornbusch, 1987, p. 98; Froot y Klemperer, 1989, p. 623). 
- El grado de asimetría (histéresis) de las decisiones de entrada o salida de las firmas de una industria determinada cuando la tasa de cambio varía (Krugman y Baldwin, 1987, p. 34; Baldwin, 1988, p. 781; Dixit, 1989, p. 220).

- El grado de intercambio entre firmas (Holmes, 1978; Goldstein y Khan, 1985), así como algunas decisiones de política comercial y cambiaria.

Línea macroeconómica: analiza los efectos inflacionarios de la transmisión de precios; la transmisión de la variación de la tasa de cambio hacia el nivel general de precios se da a través de los siguientes canales:

- Los precios de los bienes y servicios importados: en este caso el grado de transferencia depende de la elasticidad del nivel de precios domésticos ante cambios en los precios de los factores de producción. A mayor elasticidad, el grado de transferencia es mayor. El efecto de la variación depende de si el consumo intermedio de bienes importados en la producción es significativo, así como del grado de apertura. Entonces, bajo un régimen de cambio fijo, mayor influencia de la tasa de cambio, (pues las expectativas que tienen los agentes sobre las variaciones en la tasa de cambio son permanentes).

- Los precios de los bienes exportados: si los precios de las exportaciones tienen un peso significativo en el IPC, una apreciación de la tasa de cambio incrementa el precio de bienes exportados, lo que se traduce en una elevación del IPC. El grado de transferencia de la tasa de cambio depende de la estructura de mercado; bajo competencia perfecta, una variación de la tasa de cambio se convierte en una variación de igual proporción tanto en los precios medidos en moneda local como extranjera. Si existe poder de mercado, ante una apreciación, los precios de los bienes exportados medidos en moneda local se incrementan para contrarrestar las pérdidas por la reducción de precios medida en moneda extranjera, así el índice PT es incompleto.

- Los precios relativos entre transables y no transables: conocido como expenditures switching effect Salter (1959, p. 230). Dado que la devaluación produce un incremento en el precio relativo de los bienes transables, la demanda se desplaza hacia el sector de bienes no transables. El exceso de demanda producido por los bienes del sector conduce a un incremento en sus precios y luego en la inflación.

- Posición cíclica de la economía: cuando la economía se encuentra en la fase recesiva del ciclo, el efecto transferencia decrece, debido a que las firmas prefieren sacrificar márgenes de ganancia y no participación en el mercado.

- Entorno inflacionario. Taylor (2000) comprobó que en economías bajo ambientes de alta inflación, la transmisión de los movimientos en la tasa de cambio a los precios era casi inmediata; mientras que en economías bajo un ambiente estable 
y de baja inflación, las expectativas sobre el movimiento en la tasa de cambio son transitorias, por lo que se genera un bajo grado de Pass Through.

- Grado de apertura: la globalización ha llevado a que las economías abran las puertas al mundo. Este mayor dinamismo crea una fuerte competencia internacional, por lo que las firmas están dispuestas a sacrificar ganancias por adquirir mayor participación en el mercado. Ante una depreciación en la tasa de cambio, los precios varían en menor proporción, ya que las firmas no están dispuestas a perder participación en el mercado.

- Mayor credibilidad de la política económica: la credibilidad y las expectativas de inflación van de la mano. Si las acciones de la política monetaria generan confianza entre los agentes, un alza en la tasa de cambio es tomada como un efecto transitorio, y las firmas no transmiten este aumento a sus precios, lo que ocasiona un bajo grado de Pass Through.

- Cambios en la composición de bienes importados: si un país aumenta la cantidad de importaciones en bienes menos sensibles a la tasa de cambio, entonces una variación en dicha tasa no tiene un efecto proporcional en el nivel de precios ya sea en forma indirecta (si son inputs que se importan) o en forma directa (si son bienes de consumo importados).

- Rigidez de precios: los precios de exportación reaccionan en mayor medida a una depreciación que a una apreciación del tipo de cambio nominal. La explicación se encuentra en que los precios son rígidos a la baja debido a dos razones; la primera reside en que ante una depreciación del tipo de cambio, los bienes de exportación se vuelven más competitivos; de esta forma crece la cantidad demandada de dichos productos; pero si las firmas se encuentran en plena capacidad instalada, a corto plazo aumentan los precios finales, que optimizan la ganancia; la segunda razón radica en que, ante una apreciación, los exportadores no varían sus precios para no perder participación en el mercado, siempre que las firmas consideren que dicha apreciación es un efecto transitorio.

\section{REVISIÓN BIBLIOGRÁFICA}

La literatura que indaga sobre los efectos de los movimientos de la tasa de cambio en el nivel de precios, Pass Through, es profunda y extensa. Sin embargo, dichos estudios se pueden clasificar en dos enfoques distintos en abordar el tema: un enfoque sobre las asimetrías y no linealidad del índice PT, y por último, un enfoque que analiza la relación del efecto transferencia de la tasa de cambio y el régimen de política monetaria. 
Estimación de la relación entre el nivel de precios y la tasa de cambio para Colombia (1991-2006), mediante el filtro de Kalman

A continuación se expone una breve descripción bibliográfica sobre dichos enfoques; de tal forma el artículo se enfocará a la correlación que poseen el efecto del índice PT y el régimen de política monetaria por efectos del objetivo planteado para este trabajo.

\subsection{Análisis de dos etapas}

Los estudios realizados mediante este enfoque analizan las dos etapas de transmisión del efecto de transferencia de la tasa de cambio (en forma directa perturba los precios de importación, y en forma indirecta los precios domésticos) expuestas en secciones anteriores.

Campa y González (2002) analizan los países de la zona euro, bajo una muestra mensual que abarca el período 1998.1-2004.5. Mediante una estimación lineal para 30 productos, encuentran que el efecto transferencia de la tasa de cambio difiere entre países e industrias, y confluye sobre tasas a largo plazo. Los diferenciales en el grado del índice PT en los países analizados se deben a la heterogeneidad en la composición de sus importaciones totales y del grado de apertura de los países miembros respecto a los países no miembros. Estos autores encuentran que la creación de la unión monetaria en Europa trajo consigo un cambio en la composición total de importaciones, es decir, una variación de la elasticidad del nivel de precios con respecto a movimientos en la tasa de cambio, lo que provoca que el grado de transferencia de tasa cambio sea menor en los precios de consumo y mayor en los precios de costos de producción.

Por su parte, Marazzi y Sheets (2005) analizan este efecto en Estados Unidos en el período 1972-2004, con datos trimestrales. Bajo un modelo de dos variables, encuentran que la naturaleza de la competencia internacional y factores estructurales relacionados con procesos productivos influyeron de forma significativa en los precios de importación.

Sekine (2006) estudia el comportamiento del índice PT en las economías que conforman el G7. Bajo un modelo de dos variables con datos trimestrales en el período 1974-2004, evidencia que el grado del índice de transferencia en tasa de cambio pasó de 0.5 (década de 1980) a 0.2 (década de 1990) debido a un cambio en la composición de las importaciones, aumento de las importaciones chinas, mayor integración de los mercados e incremento de la competencia internacional. Por último, Rincón, Caicedo y Rodríguez (2005, p. 24) usan una muestra mensual para el período 1995-2002, y encuentran que en Colombia, a largo plazo, las elasticidades de la industria son estables, varían entre 0.1 y 0.8 , y a corto plazo estas son de 0.1 y 0.7 . 


\subsection{Asimetrías y no linealidad}

La asimetría del tipo de cambio analiza cómo las relaciones entre los movimientos del tipo de cambio y los niveles de precios varían en el tiempo y se encuentran ligados al estado imperante de la economía en el momento de ocurrir los choques cambiarios, del tamaño y del signo de los mismos.

Existen tres casos posibles de asimetría en el efecto transferencia en el tipo de cambio. Dichas asimetrías son:

- El estado imperante de la economía en el momento de ocurrir las perturbaciones cambiarias: si el producto se encuentra por encima del producto potencial, se espera un nivel alto de inflación: bajo una situación de auge, las firmas pueden transferir en forma más fácil al precio final, los costos provocados por una depreciación.

- El tamaño de la economía: existe una relación inversa entre el grado de apertura y los niveles del índice PT

- Magnitud de la perturbación en el tiempo de cambio: si la depreciación es profunda, las firmas tiene mayores incentivos para elevar sus precios.

Mendoza (2006) analiza para Venezuela las asimetrías y la no linealidad del coeficiente PT, con datos mensuales en el período 1989-2002. Mediante un modelo LSTVAR, encuentra que el grado del índice PT depende del estado de la economía en el momento en que ocurre el choque, así como del tamaño y las perturbaciones cambiarias. El efecto transferencia en tasa de cambio es un fenómeno no lineal, es decir, una depreciación tiene un mayor efecto en el grado del índice PT que una apreciación de la moneda. Winkelried (2003), por medio de un modelo de base de tipo lineal, que empleó datos mensuales para el período 1993-2002, encuentra que el coeficiente PT en Perú es un fenómeno no lineal.

Las asimetrías del efecto transferencia en tasa de cambio en Perú se deben a:

- El estado de la economía en el momento en que ocurren las perturbaciones cambiarias. Así, en la fase recesiva las firmas tienen pocos incentivos para transmitir el aumento de costos a sus precios finales, prefieren sacrificar margen de ganancia antes que perder parte de mercado.

- Magnitud y signo de perturbación: si la depreciación es muy pronunciada, las firmas tienen mayores incentivos para elevar sus precios.

- Entorno inflacionario: si la economía se encuentra en un ambiente de alta inflación, los ajustes en los niveles de precios ante variaciones en la tasa de cambio suceden casi en tiempo real. Si la economía se encuentra en un entor- 
no de baja inflación, las variaciones en la tasa de cambio son percibidas por las firmas como transitorias, por lo que las firmas se rehúsan a modificar sus precios finales.

Winkelried (2003) encuentra que el efecto transferencia en tasa de cambio presenta una tendencia decreciente en la economía peruana, fenómeno que se explica por la convergencia hacia la estabilidad monetaria en dicho país, en la última década.

García y Restrepo (2001) estiman un modelo de costo de ajuste lineal cuadrático de los precios (Linear quadratic Price adjustment Cost model, LQAC); en este modelo las firmas evalúan el costo de cambiar sus precios frente al costo de estar alejadas del precio que la firma elegiría en caso de que no existieran costos de ajuste. Este precio puede ser tomado como el "precio óptimo" o el precio de equilibrio a largo plazo, y parte del supuesto que los precios son rígidos a corto plazo. El estudio analiza las asimetrías del coeficiente PT en Chile, en el período 1986-2000, y encuentran que el impacto acumulado de un choque permanente e inesperado del tipo de cambio sobre los precios es menor que la unidad, es decir, el efecto sobre los precios es menos que proporcional a corto plazo. Además, concluyen que el grado de transferencia de la tasa de cambio decrece cuando el producto es menor que el producto potencial. Cuando la economía se encuentra bajo una recesión, el impacto de una depreciación del tipo de cambio nominal en los costos de producción se traduce en una reducción del margen de ganancia y no en un aumento en el precio final de los bienes.

En un análisis de asimetrías y no linealidades para la economía brasileña, Da Silva (2006) estudia este fenómeno para el período 1992.2-2005.2. La metodología consiste en estimar un modelo de la curva de Phillips combinado con modelos de umbral autorregresivo (TAR). Se utilizan datos trimestrales y estimaciones en dos etapas (2SLS), con variables instrumentales para las expectativas de inflación. Se estima la curva de Phillips que relaciona la inflación con la brecha de producción (output gap), expectativas de inflación, la tasa de inflación pasada, movimientos en el tipo de cambio e inflación externa. Se evalúan tres variables umbral: brecha del producto, magnitud de variaciones en la tasa de cambio nominal, medida de la volatilidad de la tasa de cambio.

Los principales resultados a que llega este autor son:

- El efecto transferencia de la tasa de cambio no es significativo cuando el régimen existente se encuentra debajo del umbral.

- La apreciación de la tasa de cambio tiene un alto grado de transferencia en tasa de cambio cuando la economía se encuentra en un ciclo de expansión 
económico, y bajo, cuando la brecha del producto se encuentra por debajo del umbral (ciclo bajo de la economía).

- Los efectos de variaciones en la tasa de cambio a corto plazo sobre la inflación son asimétricos.

- Se encuentra que ante grandes depreciaciones de la tasa de cambio, y el nivel del coeficiente PT para el siguiente trimestre es alrededor de 11\%, mientras que pequeñas apreciaciones o depreciaciones no son significativas.

- Se estima un alto grado del coeficiente PT en períodos de baja volatilidad de la tasa de cambio nominal (cerca de 80\%) y un bajo PT en períodos de poca volatilidad (7\%).

\subsection{El régimen de política monetaria}

El trabajo de Taylor (2000) es un trabajo teórico y empírico. Realiza un análisis de la economía estadounidense en dos períodos: 1960-1969 el cual se caracteriza por tener un alto nivel de inflación, y finales de 1982-1999.3, período de gran estabilidad de precios. Encuentra que el grado de PT depende de qué tan "rígida" sea la política monetaria, pues su grado de rigidez forma expectativas permanentes o transitorias ante variaciones en la tasa de cambio, lo que reduce el poder de monopolio en una economía, y sus resultados evidencian que en un ambiente estable y de baja inflación el grado del coeficiente PT es bajo.

Taylor (2000) analiza la variación de poder de mercado en una economía pequeña y abierta; argumenta que la reducción del poder de monopolio se debe a las bajas expectativas de persistencia de precios y movimientos de costos.

Las empresas (en el caso de empresas exportadoras) pueden pensar que si aumentan los precios ante una apreciación en la tasa de cambio, sus competidores pueden no hacerlo, arriesgándose a perder parte del mercado.

El modelo de Taylor es un modelo de precios escalonados, con poder de mercado. El poder de mercado depende de qué tan diferenciados sean sus productos, del nivel de sustitución, más la probabilidad de reacción de las otras firmas.

El modelo analiza variaciones en los costos, variaciones de precios de las otras firmas y variaciones de demanda.

Las firmas maximizan su beneficio a partir del costo marginal y el precio promedio de las otras firmas. El precio óptimo para la firma representativa es:

$$
x_{t}=0.125 \sum_{i=0}^{3}\left(E_{t} c_{t+i}+E_{t} p_{t+i}+E_{t} \varepsilon_{t+i} / \beta\right)
$$


El coeficiente de 0.125 implica que si los precios de las demás firmas y los costos marginales aumentan en una unidad (en los cuatro períodos), entonces $x_{t}$ se incrementa en la misma proporción. Si el aumento solo se da en los costos marginales o solo en los precios de las otras firmas, $x_{t}$ se incrementará en 0.5 unidades.

La ecuación (2) muestra que ante un aumento del costo marginal, las firmas traspasan este efecto a sus precios en función de la persistencia en la variación del costo marginal. Asimismo, si las demás firmas elevan sus precios, la firma representativa aumentará su precio de acuerdo con las expectativas que tenga sobre la persistencia del incremento de precio de las otras firmas (si se espera que esta variación sea transitoria, la firma no cambia su precio para no correr el riesgo de perder mercado).

El modelo de Taylor (2000) manifiesta la relación entre el régimen de política monetaria y el grado de Pass Through. Un régimen fijo incita a esperar que las variaciones en la tasa de cambio tengan un efecto permanente, y que en regímenes más flexibles las variaciones en la tasa de cambio sean percibidas como transitorias. De este modo, la hipótesis de Taylor (2000) argumenta que debido a que la política monetaria persigue el objetivo de un ambiente estable y bajo de inflación, las firmas, siempre que crean en las acciones de la autoridad monetaria, presentan expectativas transitorias ante movimientos de la tasa de cambio, por lo que no se traspasa la variación de los costos de producción a los precios finales, reduciéndose el efecto Pass Through en el tipo de cambio. Taylor (2000) sustentó dicha hipótesis en un análisis de los países industrializados que adoptaron un esquema de inflación objetivo.

Taylor (2000) encuentra que:

- El poder de mercado depende de las expectativas de los futuros costos y movimientos de precios de las demás firmas.

- Para una firma que importa inputs, su costo total depende de la tasa de cambio; entonces si se espera que las variaciones en la tasa de cambio sean transitorias, el resultado en el coeficiente PT es menor.

- Si el mercado tiende a ser competitivo (ya sea en ámbitos internacionales), se esperan pequeños incrementos de precios ante presiones de demanda; la variación en los precios dependerá de las expectativa que se tengan del nivel de persistencia de shocks en la demanda.

El trabajo de Taylor (2000) ha inspirado para analizar el efecto de los regímenes cambiarios en el coeficiente PT. Su hipótesis se ha verificado en un sinnúmero de trabajos, que concluyen que bajo una política monetaria creíble el poder de mercado en las firmas se reduce, en el sentido de que genera expectativas transitorias ante choques de la tasa de cambio. 
Devereux y Yetman (2002) realizan un análisis para 122 países. Parten de un modelo en el que las firmas deciden la frecuencia óptima con que cambian los precios, la cual depende del régimen de política monetaria y del ambiente inflacionario existente. Sus resultados muestran que cuanto mayor sea la incidencia de la política monetaria en el comportamiento de los precios, más baja será la frecuencia de ajuste y, por tanto, habrá un menor grado en el coeficiente PT. En su análisis para 122 países, dichos autores encuentran que la tasa de cambio es determinada en gran medida por factores macroeconómicos, en particular, la rigidez de los precios. Al igual que Taylor (2000), encuentran que el grado de transferencia en tasa de cambio es sensible al régimen de política monetaria; así una alta o baja flexibilidad de dicho régimen determina expectativas transitorias o permanentes de los agentes ante variaciones en la tasa de cambio.

Albuquerque y Portugal (2004) desarrollan un modelo teórico y empírico para Brasil en el período 1980-2002 con datos trimestrales. El modelo teórico supone competencia imperfecta, donde las firmas son tomadoras de precios en el mercado internacional, pero tienen poder de mercado en el espacio nacional. Primero estiman un modelo lineal por mínimos cuadrados con variables Dummy que permiten diferenciar el antes y después de la adopción del plan Real de Brasil (1994-1998) y la adopción del régimen de flotación libre (en 1999). Sin embargo, este modelo presenta inestabilidad en los parámetros y errores de especificación. Para corregir dichos errores utilizan el filtro de Kalman, cuya técnica consiste en permitir que los parámetros cambien con el tiempo. Por medio de esta técnica se encontró evidencia de que el ambiente inflacionario y el régimen de tipo de cambio influyeron en la reducción de los niveles de Pass Through.

El estudio de González y Gaytan y (2006) en México para el período 1992-2005 es interesante, ya que dicho país implementa en 1995 un régimen de flotación libre y una gradual adopción de inflación targeting, al igual que lo hizo Colombia en 1999. El objetivo de este trabajo es analizar las transformaciones en el mecanismo de transmisión de política monetaria, como uno de los aspectos relacionados con el cambio del comportamiento de la inflación en el período de análisis. Mediante un modelo de Markov Switching de vectores autorregresivos identifica los períodos en que se registran los cambios estructurales y analiza la relación entre las principales variables, las cuales han cambiado a través del tiempo.

González y Gaytan (2006, p. 33) concluyen que la adopción formal del esquema de inflación objetivo en el año 2001 llevó a un importante cambio estructural en el mecanismo de transmisión de la política monetaria. Tras dicho cambio, las variaciones en el tipo de cambio han tenido menor efecto sobre el proceso de formación de precios, es decir, tienen una pequeña incidencia tanto en la tasa de inflación 
contemporánea como en la esperada. Por tanto, la tasa de interés nominal responde en menor medida ante variaciones en el tipo de cambio.

Por otra parte, Rojas, Rodríguez y Patiño (2006) en un análisis para la economía de Paraguay, bajo una muestra que abarca el período 1994-2005, mediante la técnica del Rolling Model encuentran que el nivel del coeficiente PT depende del estado de la economía y del ambiente inflacionario, del tiempo y del período empleado de la inflación (mensual, trimestral, anual). Así, si el modelo se estima con la serie de inflación interanual, el coeficiente PT es mucho más inestable que el estimado con la serie inflación mensual, pero, a su vez, en mayor medida, lo es la inestabilidad cambiaria del país.

Nkunde (2006) estudia el efecto transferencia en tasa de cambio en Tanzania en el período 1990-2005. Este emplea un modelo VAR, captura el impacto de los shocks de la tasa de cambio en la inflación. El autor percibe que aunque la economía se encontraba en un período de gran depreciación en la moneda, este fenómeno no se tradujo en un nivel de precios más altos. Al contrario, se evidenció un grado bajo en el coeficiente PT de la tasa de cambio a la inflación, debido a las reformas estructurales que se dieron en 1995, como una activa intervención del Banco Central en el control de la oferta monetaria. El estudio muestra, además, un gran impacto del crecimiento de la competencia, alta productividad y una política monetaria más rígida (que ha ocasionado bajas expectativas de inflación entre los agentes).

\section{LA POLÍTICA MONETARIA EN COLOMBIA}

En 1991 la nueva Constitución Política proclamó la independencia del Banco de la República, en el sentido de independencia política y económica. Es decir, el Banco Central puede definir sus objetivos primordiales en implementar y emplear los instrumentos para el logro de los objetivos (Clavijo, 2000). En Colombia el objetivo principal del Banco Central es el control de la inflación, en un marco de coordinación con el ejecutivo, quien tiene como objetivos el crecimiento y la generación de empleo.

Entre 1991-1994, la meta intermedia de la política monetaria en Colombia eran los agregados monetarios bajo una tasa de cambio fija con devaluación gradual. La acumulación de reservas de los años ochenta del siglo veinte, así como la entrada de capitales y la mayor liquidez en la economía, los efectos de la apertura y liberación financiera que se dieron desde 1991, llevaron a un crecimiento del consumo y del PIB. Según Correa (2004, p. 18), este crecimiento fue producto de una expansión monetaria y entrada de flujos de capital, lo cual se ilustra en los gráficos 1 y 2. 
En 1994 se adopta un esquema de bandas cambiarias, que permitió un poco la flexibilización del régimen cambiario. Entre 1996 y 1997 las medidas de política monetaria se orientaron a menores tasas de interés, mayor flujo de dinero y mayor gasto público, lo que ocasionó entre 1998 y 2000 una caída en el stock de reservas, la cual también se vio afectada por la crisis internacional. Dichas medidas condujeron a un aumento del nivel general de precios.

El primer precio afectado fue la tasa de cambio. El exceso de liquidez se canalizó en una mayor demanda por dólares, así la tasa de cambio pasó del piso al techo de la banda cambiaria en septiembre de 1997. Fenómeno que al comienzo se consideró positivo para las exportaciones y la cuenta corriente, pero que se hizo insostenible lo que a la postre llevó a una recesión económica del país en 1999. En la época de recesión la política monetaria fue contractiva y el aumento de la tasa de interés se tradujo en una disminución del consumo, como se observa en los gráficos 1 y 2.

A partir del año 2000 se implementó como estrategia monetaria la inflación objetivo, que ha llevado a menores tasas de interés y menores tasas de inflación. La flotación de la tasa de cambio permite que la autoridad monetaria pueda utilizar la política monetaria como herramienta de estabilización económica más efectiva (Clavijo, 2000).

Grafico 1: Tasa de interés real pasiva de Colombia 1994-2003

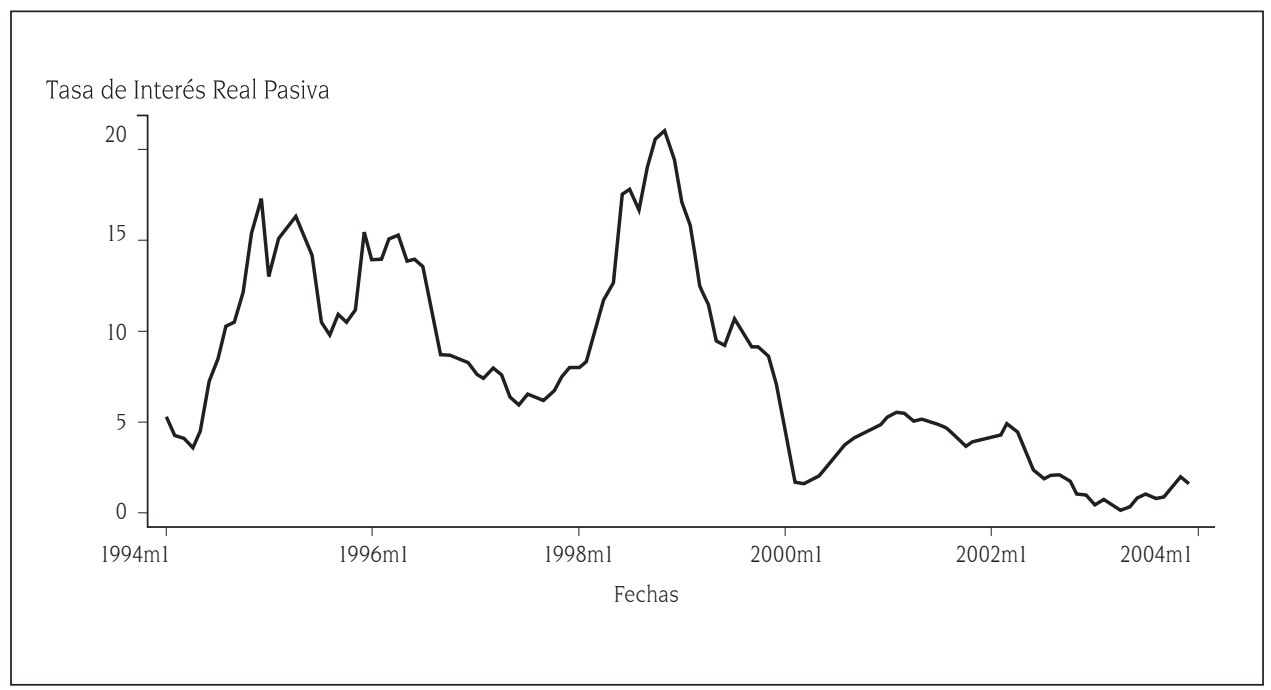

Fuente: elaboración propia con información del Banco de la Republica. 
Gráfico 2. Variación de Consumo Colombia 1994-2003

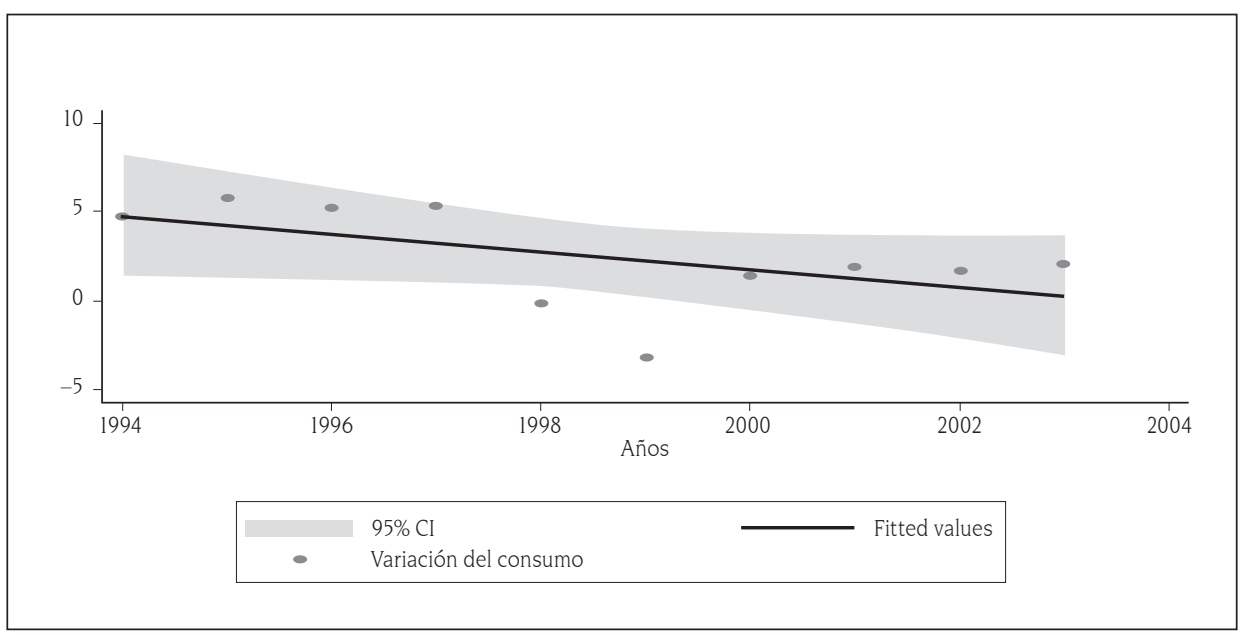

Fuente: elaboración propia con información del Banco de la Republica

\subsection{Régimen de bandas cambiarias (1994-septiembre 1999)}

A comienzos de 1991, de acuerdo con Urrutia (2002, p. 21), Colombia se consideraba como un país atractivo para la inversión extranjera, debido a hallazgos petroleros que permitían garantizar estabilidad cambiaria en una senda de mediano plazo. La entrada de flujos de capital al país aumentó de forma notable. El Banco de la República trató de compensar este fenómeno mediante la compra de reservas por medio de operaciones de mercado abierto. Sin embargo, por mantener bajo control los medios de pago las subastas se negociaban a altas tasas de interés, cuyo efecto era un mayor aumento en la entrada de capitales.

La entrada de capitales siguió en ascenso a pesar de las medidas tomadas por el Banco de la República (como asignar plazo a los certificados de cambio) y estas no mostraban los resultados esperados. Así la junta directiva decide flexibilizar más la tasa de cambio, mediante la creación del sistema de bandas cambiarias.

Pasar de un régimen de tasa de cambio fijo (minidevaluaciones diarias) al sistema de bandas cambiarias fue un paso muy criticado, en especial por el gremio de los exportadores, que consideraban que el sistema de bandas cambiarias solo traería problemas de revaluación.

Las bandas cambiarias contribuyeron a un mayor control de los agregados monetarios y del crédito, el cual venía en aumento. La crisis asiática afectó los términos de intercambio y el flujo de capitales en Colombia. La junta directiva decide devaluar la banda cambiaria en septiembre de 1998, ya que se consideraba 
que dicha crisis había cambiado el equilibrio de la tasa de cambio. La crisis rusa intensificó aún más la disminución en la entrada de capitales, razón por la cual la banda cambiaria se devalúa de nuevo.

\subsection{La recesión de 1999}

Para Urrutia (2002, p. 16) la crisis de 1999 se explica porque se presentó la siguiente situación en aquel momento de la historia económica:

En el primer trimestre de 1998 existía un déficit en la cuenta corriente de la balanza de pagos de $8 \%$ del PIB, y que a raíz de las crisis asiática y rusa el mercado internacional dejó de financiar ese déficit. En el segundo trimestre de 1999 la cuenta corriente estaba en equilibrio. Esto quiere decir que en el corto plazo, 15 meses, el gasto en la economía tuvo que caer $8 \%$ del PIB.

La caída de los flujos de capital y el encarecimiento del endeudamiento externo contribuyeron a la devaluación del tipo de cambio, lo que redujo la cantidad demandada de bienes importados y por esta vía se contrajo la demanda agregada, que se vio afectada por el aumento de las tasas de interés debido a la elevación de la prima de riesgo.

En este período, la política monetaria fue restrictiva en 1998 y expansionista a partir de ese año. En diciembre de 1997 la junta estableció la meta de crecimiento de la base monetaria en 16\% para 1998, pero dada la venta de reservas, la base ajustada terminó con un crecimiento de 8.1\%. En diciembre de 1998 se estableció el corredor monetario de 1999 con un crecimiento de 28.4\%, y la base ajustada creció 24.83\%. De acuerdo con Urrutia (2002, p. 17), el PIB nominal de Colombia, pasó del $15.4 \%$ en 1998, al 7.9\% en 1999 lo que se encuentra por debajo del crecimiento de la oferta monetaria.

La banda cambiaria se eliminó en medio de un ambiente de crisis económica internacional, salidas de capitales y fuertes ataques especulativos, y un intenso aumento en el gasto público, lo que afectó de forma negativa la credibilidad de la banda cambiaria. Bajo el esquema de agregados monetarios no se evitó la sobrevaloración cambiaria, no fue consistente con la reducción de la inflación y no construyó suficiente credibilidad.

\subsection{Inflación objetivo (1999.4-2004)}

El 25 de septiembre de 1999 se decide pasar a un sistema de libre flotación del tipo de cambio nominal. Bajo un esquema de inflación objetivo, el banco interviene para establecer un sendero a las tasas mediante operaciones compra o venta de reservas de acuerdo con el Informe adicional de la Junta Directiva del Banco de la 
Republica al Congreso (BR, 1999, p. 16). Tal como describe Zuluaga (2004, p. 4) la política monetaria de la autoridad monetaria de Colombia estable que:

La junta directiva del banco fija anualmente una inflación objetivo y establece valores de referencia para el nivel de los agregados monetarios compatibles con los objetivos para el crecimiento del producto, con el nivel de déficit fiscal y otras variables económicas

Desde la adopción de este esquema, la inflación se ha reducido a un dígito. Se ha generado confianza y credibilidad entre los agentes, debido al cumplimiento de las metas de inflación y a la transparencia de las medidas.

\section{ANÁLISIS EMPÍRICO}

La hipótesis de Taylor (2000) argumenta que cuando se pasa a un régimen monetario más flexible y creíble, las expectativas de los agentes ante la persistencia de variaciones de la tasa de cambio se reducen y ven dichos choques solo como efectos transitorios, que reducen el nivel del coeficiente de PT en la economía.

Dado el cambio de régimen que se dio en septiembre de 1999 en Colombia, cuando se eliminan las bandas cambiarias y se pasa a un régimen de flotación más flexible (flotación administrada) bajo un esquema de inflación objetivo, se quiere probar la hipótesis de Taylor (2000) y analizar si dicho cambio llevó a un cambio estructural, que podría afectar la relación entre el tipo de cambio y la inflación.

Se estudia la relación entre los movimientos en el tipo de cambio y su efecto en el nivel de precios, mediante dos indicadores: el Índice de Precios al Consumidor (IPC) y el Índice de Precios al Productor (IPP), a fin de indagar si existen diferencias en el nivel del coeficiente PT de acuerdo con la medida de inflación que se utilice.

\subsection{Datos}

El período de análisis corresponde a 1991-2006. Se utilizan datos trimestrales. Todas las variables se desestacionalizaron a través de la metodología X12 ARIMA y fueron calculadas como la primera diferencia de su transformación logarítmica, con el fin de obtener variables estacionarias. La prueba de raíz unitaria muestra que todas las variables, con excepción de las expectativas (Expect) son integradas de orden uno. Las variables utilizadas fueron:

PPD: Índice de Precios al Productor de Colombia. Incluye bienes producidos y consumidos y los importados. Total nacional.

IPD: $\quad$ Índice de Precios al Consumidor de Colombia. Total nacional.

TC: $\quad$ Tasa de cambio bilateral promedio, Colombia-Estados Unidos. 
OPEN: Representa el grado de apertura de la economía. Calculado como la sumatoria del total de importaciones y el total de exportaciones sobre el PIB. Se espera una relación negativa de esta variable con los precios.

Gap: Desviación del PIB de su nivel potencial. La tendencia de la serie fue extraída mediante el filtro de Hodrick Prescott. Gap es la diferencia entre el valor observado del PIB, expresado en logaritmos, y la tendencia calculada por el filtro de Hodrick Prescott, como una aproximación de la desviación del PIB con respecto a su nivel potencial. Se espera un signo positivo de esta variable. Si el PIB está por debajo de su nivel, es decir, la economía se encuentra en un ciclo recesivo, el nivel de precios cae al igual que el Gap. Si la economía se encuentra en una fase expansiva, es decir, el producto está por encima de su nivel, un incremento del PIB lleva a un incremento del nivel de precios.

Expect: Variable que captura las expectativas de los agentes sobre la inflación. Las expectativas pueden ser adaptativas o racionales. Se estimó un coeficiente de error estándar para cada clase de expectativa. Dichos coeficientes son 0.56 para expectativas adaptativas y 0.44 para expectativas racionales. Así, para el período 1991-junio 2003, la variable expectativa corresponde a expect $_{t}=\min \sum \sqrt{\left(e_{t+1}-e_{t-1}\right)^{2}}$, donde expect es la variación mensual del IPC. A partir de septiembre de 2003 se utilizaron los datos sobre la encuesta de expectativas, que realiza el Banco en forma trimestral a partir de septiembre de 2003.

\subsection{Modelo lineal}

El primer paso fue realizar un análisis de regresión múltiple por medio de mínimos cuadrados ordinarios. El modelo a estimar fue:

$$
p=\mu+\alpha_{1} t c_{t-1}+\alpha_{2} \text { open }_{t-1}+\alpha_{3} \text { gap }_{t-1}+\alpha_{4} \text { expect }_{t-1}+\varepsilon_{t}
$$

Donde P representa el IPC y el IPP para cada caso. Los dos modelos presentan errores de especificación (Reset Test), problemas de inestabilidad ya sea en los parámetros y/o en las varianzas y signos incorrectos (modelo con IPC como variable independiente). Dados estos resultados y con los cambios ya conocidos que se dieron en septiembre de 1999 en materia de política monetaria, se utilizan variables Dummy en los coeficientes de la tasa de cambio, para introducir el cambio de régimen ocurrido en ese mes.

\section{Modelos lineales con variables Dummy}

Para reflejar el cambio de régimen que se dio en septiembre de 1999 en Colombia, se utiliza una variable Dummy para verificar si dicho cambio tuvo efectos 
Estimación de la relación entre el nivel de precios y la tasa de cambio para Colombia (1991-2006), mediante el filtro de Kalman

significativos sobre los parámetros en el período analizado. De este modo, el modelo a estimar es:

$$
p=\mu+\left(\alpha_{1}+\alpha_{11} d_{11}\right) t c_{t-1}+\alpha_{2} \text { open }_{t-1}+\alpha_{3} \text { gap }_{t-1}+\alpha_{4} \text { expect }_{t-1}+\varepsilon_{t}
$$

Donde P representa el IPC y el IPP para cada caso. La variable $\mathrm{d}_{11}$ representa el período después de que se adopta la inflación objetivo, a partir de valores iguales a uno después del cuarto trimestre de 1999. Entonces, $\alpha_{11}$ es el coeficiente de la pendiente diferencial. Muestra qué tanto difiere el coeficiente de la pendiente de $\mathrm{P}$ en el segundo período, donde $\mathrm{d}_{11}$ es igual a uno. $\alpha_{11} \mathrm{tc}_{\mathrm{t}-1}$ permite diferenciar los coeficientes de los dos períodos.

Las regresiones de las submuestras se definen como:

Período 1991.1-1999.3 (régimen de bandas cambiarias)

$$
E\left(p_{t} / d_{t}=0, t c_{t-1}\right)=\mu+\alpha_{11} t c_{t-1}+\alpha_{2} \text { open }_{t-1}+\alpha_{3} \text { gap }_{t-1}+\alpha_{4} \text { expect }_{t-1}
$$

Período 1999.4-2006.4 (régimen de flotación administrada)

$$
E\left(p_{t} / d_{t}=1, t c_{t-1}\right)=\mu+\left(\alpha_{1}+\alpha_{11}\right) t c_{t-1}+\alpha_{2} \text { open }_{t-1}+\alpha_{3} \text { gap }_{t-1}+\alpha_{4} \text { expect }_{t-1}
$$

La inclusión de las variables Dummy corrigió los problemas de estabilidad en el modelo. Sin embargo, las variables open, gap y expect resultaron no ser significativas en ninguno de los dos modelos estimados. $\left(\alpha_{1}+\alpha_{11}\right)$ es el coeficiente de PT para el período 1999.4 - 2006.1. Sin embargo, no se puede rechazar Ho: $\left(\alpha_{1}+\alpha_{11}\right)=0$, en ninguno de los dos modelos. El no rechazo de dicha hipótesis significa que el efecto transferencia de la tasa de cambio sobre los precios después de 1999 es nulo, lo cual es una contradicción económica. En la estimación efectuada con variables Dummy, el test de Ramsey evidencia mala especificación en el modelo. La razón se puede encontrar en que realizar un análisis en este período bajo un modelo con parámetros invariantes en el tiempo no es el procedimiento más adecuado, y más aún si se considera la transformación del régimen cambiario, cuyo cambio de estructura pudo llevar a redefinir el coeficiente de PT.

Alburquerque y Portugal (2004) encuentran que al utilizar mínimos cuadrados, las variables Dummy son incapaces de identificar cambios estructurales en la política monetaria, debido a que se trabaja con parámetros que no varían en el tiempo. La solución es estimar el modelo con parámetros que cambien en el tiempo, es decir, emplear el filtro de Kalman. 


\subsection{Análisis del filtro de Kalman}

El filtro de Kalman se aplicó mediante un proceso general. Primero se probó un modelo en que todos los coeficientes se consideraban estocásticos. Las ecuaciones de estado y las ecuaciones de actualización son:

Ecuación de estado:

$$
P d=\mu_{t}+\alpha_{1, t} e_{t-1}+\alpha_{2, t} \text { open }_{t-1}+\alpha_{3, t} \text { gap }_{t-1}+\alpha_{4, t} \text { expect }_{t-1}+\varepsilon_{t}
$$

Ecuaciones de medida

$$
\begin{aligned}
& \mu_{t}=c_{1}+c_{2} \mu_{t-1}+\vartheta_{\mu, t} \\
& \alpha_{1, t}=a_{11}+a_{12} \alpha_{1, t-1}+a_{13} d_{11}+\vartheta_{\alpha 1, t} \\
& \alpha_{2, t}=a_{21}+a_{22} \alpha_{2, t-1}+\vartheta_{\alpha 2, t} \\
& \alpha_{3, t}=a_{31}+a_{32} \alpha_{3, t-1}+\vartheta_{\alpha 3, t} \\
& \alpha_{4, t}=a_{41}+a_{42} \alpha_{4, t-1}+\vartheta_{\alpha 4, t}
\end{aligned}
$$

El número de variables estocásticas se redujo de acuerdo con la significancia de la varianza en la ecuación de estado y con el criterio de Akaike y Schwartz. Los resultados determinaron que la variable de la tasa de cambio y el intercepto se podían tomar como coeficientes estocásticos.

Es importante tener en cuenta la estructura de una Representación de Espacios de Estado (State Space). Si los vectores de estado se restringen a una caminata aleatoria (Random walk), esto implica que las perturbaciones de los coeficientes estocásticos son permanentes; si se trabaja en un formato auto-regresivo de orden uno Ar (1) aunque las perturbaciones de los coeficientes estocásticos son persistentes, se consideran efectos transitorios (Alburquerque y Portugal, 2004).

Los choques en la tasa de cambio sobre el nivel de precios no son permanentes, sino que afectan los precios en diferentes grados. Por lo que trabajar bajo un formato $\operatorname{Ar}(1)$, es recomendable.

La variable Dummy se introdujo en las ecuaciones de actualización, para verificar si el cambio de régimen afectó el nivel de precios. Así, $\mathrm{d}_{11}$, asume el valor cero para el subperíodo 1991.1-1999.3 y valores iguales a uno en el subperíodo 1999.4-2006.4.

\subsubsection{Estimación del filtro de Kalman con IPC como variable dependiente}

El modelo estimado es: 


$$
\begin{aligned}
& \text { Ipc }=\mu_{t}+\alpha_{1, t} e_{t-1}+\alpha_{2, t} \text { open }_{t-1}+\alpha_{3, t} \text { gap }_{t-1}+\alpha_{4, t} \text { expect }_{t-1}+\varepsilon_{t} \\
& \mu_{t}=c_{1}+c_{2} \mu_{t-1}+\vartheta_{\mu, t} \\
& \alpha_{1, t}=a_{11}+a_{12} \alpha_{1, t-1}+a_{13} d_{11}+\vartheta_{\alpha 1, t} \\
& \alpha_{2, t}=\alpha_{2, t-1} \\
& \alpha_{3, t}=\alpha_{3, t-1} \\
& \alpha_{4, t}=\alpha_{4, t-1}
\end{aligned}
$$

En la tabla 1 se muestran los resultados obtenidos. La variable Gap $\left(\alpha_{3}\right)$ no es significativa y no presenta el signo esperado. Mientras que la variable Open $\left(\alpha_{2}\right)$ y la variable Expect $\left(\alpha_{4}\right)$ son significativas y presentan el signo esperado.

El coeficiente de la varianza tanto del intercepto como de la tasa de cambio $\left(\zeta_{\mu_{\mu, t^{\prime}}} \zeta \alpha_{1 t}\right)$ son significativos. Los coeficientes estimados varían en el tiempo y el filtro de Kalman captura dichos cambios en los coeficientes. Es decir, se tiene una buena estimación por medio de este filtro, ya que en una estimación por mínimos cuadrados los parámetros son constantes.

El coeficiente $C_{1}$ resultó ser significativo, mientras que $C_{2}$ no lo es. Este resultado implica que la media del intercepto no es nula y los choques sobre el nivel de precios tienen efectos transitorios. (Ver tabla 1).

En cuanto a los coeficientes de PT, $\mathrm{a}_{11}$ y $\mathrm{a}_{12}$ resultaron ser significativos. Es decir, el coeficiente de PT es importante para explicar la dinámica de los precios en el período analizado, los choques en la tasa de cambio son persistentes pero con efectos transitorios. El pronóstico para el efecto de transferencia en tasa de cambio para el período $t+1$ corresponde al valor medio $a_{11}$, es decir, un incremento en $1 \%$ en la tasa de cambio causa en promedio un incremento de $0.019 \%$ en el nivel general de precios. Finalmente, $\mathrm{a}_{13}$ es significativo, implica que el régimen cambiario afecta la dinámica de los niveles del coeficiente PT.

Los valores de los coeficientes del filtro de Kalman, para el caso del IPC como variable dependiente, en el período 1991.1-1999.3 el valor medio de PT fue 0.028, mientras que para el período 1999.4-2006.1 en promedio el efecto transferencia fue de solo 0.023 .

\subsubsection{Estimación del filtro de Kalman con IPP como variable dependiente}

La ecuación estimada en este caso es: 
Fernando Chavarro Miranda - Manfred Grautoff Laverde - Erika Viviana Mancipe Moncada

Tabla 1. Filtro de Kalman variable dependiente Índice Precios Consumidor.

\begin{tabular}{|c|c|c|c|c|}
\hline Variable & Coeficientes & Error Estándar & $\mathrm{T}$-Estadístico & P-valor \\
\hline \multicolumn{5}{|c|}{ Ecuación de medida } \\
\hline$\alpha 2$ & $-1,341,353$ & 0.582548 & $-2,302,56$ & 0.0213 \\
\hline$\alpha 3$ & -0.074142 & 0.242225 & -0.306087 & 0.7595 \\
\hline$\alpha 4$ & 0.022565 & 0.005123 & $4,404,811$ & 0.0000 \\
\hline \multicolumn{5}{|c|}{ Intercepto - Ecuación de Estado } \\
\hline $\mathrm{Cl}$ & 0.019595 & 0.004967 & $3,944,952$ & 0.0001 \\
\hline $\mathrm{C} 2$ & 0.003831 & 0.018056 & 0.212206 & 0.8319 \\
\hline$\zeta \mu, \mathrm{t}$ & -5.83212 & 0.416824 & -7.56065 & 0.000 \\
\hline \multicolumn{5}{|c|}{ Coeficiente de PT - Ecuación de estado } \\
\hline all & 0.019884 & 0.007424 & 2.907419 & 0.0000 \\
\hline al2 & 0.990477 & 0.024548 & 4.034927 & 0.0000 \\
\hline al3 & 0.031750 & 0.013560 & $2,341,405$ & 0.0192 \\
\hline$\zeta \alpha 1, \mathrm{t}$ & -2.3054 & 0.21252 & -4.9296 & 0.035 \\
\hline Log likelihood & $1,356,486$ & \multicolumn{2}{|c|}{ Akaike info criterion } & $-3,957,769$ \\
\hline Schwarz criterion & $-3,654,177$ & \multicolumn{2}{|c|}{ Hannan-Quinn criter. } & $-3,838,169$ \\
\hline
\end{tabular}

Fuente: elaboración propia.

$$
\begin{aligned}
& \text { Ipp }=\mu_{t}+\alpha_{1, t} e_{t-1}+\alpha_{2, t} \text { open }_{t-1}+\alpha_{3, t} \text { gap }_{t-1}+\alpha_{4, t} \text { expect }_{t-1}+\varepsilon_{t} \\
& \mu_{t}=c_{1}+c_{2} \mu_{t-1}+\vartheta_{\mu, t} \\
& \alpha_{1, t}=a_{11}+a_{12} \alpha_{1, t-1}+a_{13} d_{11}+\vartheta_{\alpha 1, t} \\
& \alpha_{2, t}=\alpha_{2, t-1} \\
& \alpha_{3, t}=\alpha_{3, t-1} \\
& \alpha_{4, t}=\alpha_{4, t-1}
\end{aligned}
$$

En la tabla 2 se muestran los resultados obtenidos. En este caso las variables Open $\left(\alpha_{2}\right)$, Gap $\left(\alpha_{3}\right)$ y Expect $\left(\alpha_{4}\right)$ son significativas y presentan el signo esperado.

El análisis de los resultados es similar al del caso anterior. Al utilizar IPP como variable dependiente, muestra que los coeficientes actuales varían en el tiempo $\left(\zeta_{\mu, t}\right.$ y $\zeta \alpha_{1, t}$ son significativos). Los coeficientes del PT indican que este es importante 
Estimación de la relación entre el nivel de precios y la tasa de cambio para Colombia (1991-2006), mediante el filtro de Kalman

Tabla 2. Filtro de Kalman Variable dependiente: Índice Precios Productor

\begin{tabular}{|c|c|c|c|c|}
\hline Variable & Coeficientes & Error Estándar & $\mathrm{T}$ - Estadístico & $\mathrm{P}$-valor \\
\hline \multicolumn{5}{|c|}{ Ecuación de medida } \\
\hline$\alpha_{2}$ & $-1,807,11$ & 0.498384 & $-3,625,94$ & 0.0003 \\
\hline$\alpha_{3}$ & 0.423700 & 0.207012 & $2,046,743$ & 0.0407 \\
\hline$\alpha_{4}$ & 0.022652 & 0.004284 & $5,287,586$ & 0.0000 \\
\hline \multicolumn{5}{|c|}{ Intercepto - Ecuación Estado } \\
\hline $\mathrm{C}_{1}$ & 0.013686 & 0.003907 & $3,503,262$ & 0.0005 \\
\hline $\mathrm{C}_{2}$ & 0.003777 & 0.016783 & 0.225030 & 0.8220 \\
\hline$\zeta_{\mu, \mathrm{t}}$ & -5.1754 & 0.43252 & -12.4785 & 0.000 \\
\hline \multicolumn{5}{|c|}{ Coeficiente de PT - Ecuación de estado } \\
\hline$a_{11}$ & 0.017587 & 0.006158 & $2,394,348$ & 0.0000 \\
\hline$a_{12}$ & 0.996964 & 0.023891 & $4,173,031$ & 0.0000 \\
\hline$a_{13}$ & 0.017560 & 0.009207 & $1,907,214$ & 0.0565 \\
\hline$\zeta \alpha_{1, t}$ & -1.87824 & 0.126432 & -3.7659 & 0.013 \\
\hline Log likelihood & $1,447,306$ & \multicolumn{2}{|c|}{ Akaike info criterion } & $-4,241,580$ \\
\hline Schwarz criterion & $-3,937,987$ & \multicolumn{2}{|c|}{ Hannan-Quinn criter. } & $-4,121,979$ \\
\hline
\end{tabular}

Fuente: elaboración propia.

para entender la dinámica de los precios, los choques sobre la tasa de cambio son persistentes pero no permanentes y el cambio de régimen afectó la dinámica de los niveles de transferencia en tasa de cambio.

En este caso, un incremento de $1 \%$ de la tasa de cambio incrementa en promedio en 0.017 el nivel de precios. Entre el período 1990.1-1999.3 el promedio del coeficiente de PT fue de 0.027, mientras que para el período 1999.4 -2006.1 fue de sólo 0.023 .

\section{CONCLUSIONES}

En septiembre de 1999 la junta directiva del Banco de la República decidió abandonar las bandas cambiarias debido a la falta de credibilidad en estas, y adoptó un tipo de cambio flexible pero con intervención bajo una estrategia de inflación objetivo. Este cambio estructural y la recesión de 1999 originaron la desinflación, que llevó la misma a un dígito, lo que permitió dar consistencia temporal a la política monetaria. Así los agentes tienen expectativas transitorias frente a choques en la tasa de cambio que modifican el nivel de precios, y el efecto transferencia se reduce. 
La baja relación entre expectativas y regímenes cambiarios flexibles se denomina, en la literatura económica, hipótesis de Taylor.

Mediante el filtro de Kalman se encontró que el coeficiente PT se redujo entre los años 1999-2006 debido al cambio de régimen. El primer modelo (IPC como variable dependiente) refleja un valor medio de 0.028 para el primer período (1991-2006) y un valor medio de 0.0236 para el segundo período (1999-2006). El segundo modelo (IPP como variable dependiente) coincide con la disminución del efecto PT dado el cambio de régimen. Así, para el primer período, el valor medio es de 0.024 y en el segundo período, el valor medio es 0.023 .

La diferencia de coeficientes de pronóstico de transferencia en tasa de cambio no es sensible entre los modelos estimados. El segundo modelo indica un coeficiente de 0.017 , mientras que el primero muestra un coeficiente de 0.019. Es decir, el coeficiente no varía de forma significativa si se utiliza IPC o IPP como indicador del nivel general de precios.

Los investigadores de fenómenos cambiarios y monetarios podrían analizar los efectos de cambios estructurales sobre la dinámica de los niveles de Pass Through, con modelos que estimen parámetros que cambian en el tiempo, para lograr identificar los cambios de política monetaria que inciden sobre el nivel Pass Through. Una estimación por medio de mínimos cuadrados ordinarios no tiene capacidad de reflejar dichos cambios y conduce a conclusiones equivocadas a causa de la mala especificación del modelo.

\section{BIBLIOGRAFÍA}

Albuquerque, C. R. y Portugal, Marcelo Savino (2004). Pass-through from exchange rate to prices in Brazil: an analysis using time-varying parameters for the 1980-2002 periods. En: Revista de economía, Montevideo, Vol. 12, No. 1, p. 17-73.

Banco de la República (1999). Informe adicional de la Junta Directiva al Congreso de la República, octubre, Bogotá.

Baldwin, R. (1988). Hysteresis in import prices: the beachhead effect. En: The American Economic Review, Vol. 78, No. 4, p. 773-785.

Campa, José Manuel y González Mínguez, José M. (2002). Differences in exchange rate passthrough in the euro area. En: European Economic Review, Elsevier, Vol. 50, No 1, p. 121-145.

Clavijo, S. (2000). Política monetaria y cambiaria en Colombia: progresos y desafíos (1991-2002). En: Borradores de Economía, No 201, Banco de la República.

Correa Ospina, Alexander (2004). Canales de transmisión monetaria: una revisión para Colombia. En: Economía, Gestión y Desarrollo. Universidad Javeriana, No. 2, septiembre, p. 9-31.

Dixit, Avinash ( 1989). Hysteresis, import penetration and exchange rate pass through. En: The Quarterly Journal of Economics, Vol. 104, No. 2, p. 205-228. 
Estimación de la relación entre el nivel de precios y la tasa de cambio para Colombia (1991-2006), mediante el filtro de Kalman

Da Silva, A Minilla (2006). Nolinear mechanism of the exchange rate pass through: a Phillips curve model with threshold for Brazil. En: Working paper Series, No. 122, Banco Central do Brazil.

Devereux, M. y Yetman, J. (2002). Price- setting and exchange rate pass- through: Theory and evidence. Hong Kong Institute for Monetary Research Working Paper, No. 22, diciembre.

Dornbusch, Rudiger (1987). Exchange rates and prices. En: American Economic Review, American Economic Association, Vol. 77, No. 1, p. 93-106.

Froot, Kenneth A. y Klemperer, Paul D, (1989). Exchange Rate Pass-Through When Market Share Matters. En: American Economic Review, American Economic Association, Vol. 79, No. 4, p. 637-654.

García, C. y Restrepo, J. (2001). Price inflation and exchange rate Pass Through in Chile. En: Working Papers Central Bank of Chile, No 128, Central Bank of Chile.

Goldstein, M. y M.S. Khan (1985). Income and price effects in foreign trade. In R.W. Jones and P.B. Kenen (eds.), Handbook of International Economics, Vol. 2, Elsevier Science Publishers B.V.

González García, Jesús y Gaytan, Alejandro (2006). Structural Changes in the Transmission Mechanism of Monetary Policy in Mexico: A Non-linear VAR Approach. Banco de Mexico, Working Paper, No. 06, abril.

Holmes, P. (1978). Industrial Pricing Behavior and Devaluation, Macmillan, London.

Krugman, Paul (1987). The narrow moving band, the Dutch disease, and the competitive consequences of Mrs. Thatcher: Notes on trade in the presence of dynamic scale economies. En: Journal of Development Economics, Elsevier, Vol. 27, No. 1-2, p. 41-55.

Krugman, Paul (1999). The Return of Depression Economics, Allen Lane, London and Norton, New York.

Krugman, Paul R. y Baldwin, Richard E. (1987). The Persistence of the U.S. Trade Deficit. En: Brookings Papers on Economic Activity, Economic Studies Program, The Brookings Institution, Vol. 18 No. 1, p. 1-56.

Marazzi, M. y Sheets, N. (2005). Decline exchange rate Pass Through to U.S. import prices: the potential role of global factors", Board of Governors of the Federal Reserve System International Finance Discussion Papers Number 833.

Mendoza Lugo, Omar A. (2006). Las asimetrías del Pass-Through en Venezuela. En: Revista Monetaria, Vol. XXIX, No. 2, abril- junio.

Nkunde, Mwase (2006). An Empirical Investigation of the Exchange Rate Pass-Through to Inflation in Tanzania, IMF Working Papers number 06/150, International Monetary Fund.

Rincón, H.; Caicedo, E. y Rodríguez, N. (2005). Exchange rate Pass Through effects: a disaggregate analysis of Colombian imports of manufactured goods. En: Borradores de Economía, No. 330, Banco de la Republica.

Rojas, B.; Rodríguez, N. y Patiño, M. J. (2006). Estimación del efecto Pass Through para la economía paraguaya. En: Documentos de trabajo, No. 09, Gerencia de Estudios Económicos del Banco Central del Paraguay. 
Salter, W., 1959, Internal and External Balance: The Role of Price and Expenditure Effects. En: Economic Record, Vol. 35, p. 226-238.

Sekine, Toshitaka (2006). Time-varying exchange rate Pass-Through: experiences of some industrial countries. Bank for International Settlements in its series Papers, No. 202.

Taylor, J. (2000). Low Inflation, Pass-Through, and the Pricing Power of Firms. En: European Economic Review, No. 44.

Urrutia, Miguel (2002). Una visión alternativa: la política monetaria y cambiaria en la última década. En: Borradores de Economía, Banco de la República.

Winkelried, Diego (2003). Es asimétrico el Pass Through en el Perú, Banco Central de Reservas del Perú.

Zuluaga, Blanca (2004). La política monetaria: teoría y caso colombiano. En: Apuntes de Economía, No. 1, Universidad Icesi, 27p. 